\title{
A Characterization of the Brightness Oscillations During Thermonuclear Bursts From 4U 1636-536
}

\author{
M. Coleman Miller \\ Department of Astronomy and Astrophysics, University of Chicago \\ 5640 South Ellis Avenue, Chicago, IL 60637, USA \\ miller@bayes.uchicago.edu
}

\begin{abstract}
The discovery of nearly coherent brightness oscillations during thermonuclear X-ray bursts from six neutron-star low-mass X-ray binaries has opened up a new way to study the propagation of thermonuclear burning, and may ultimately lead to greater understanding of thermonuclear propagation in other astrophysical contexts, such as in Type Ia supernovae. Here we report detailed analyses of the $\sim 580 \mathrm{~Hz}$ brightness oscillations during bursts from $4 \mathrm{U}$ 1636-536. We investigate the bursts as a whole and, in more detail, the initial portions of the bursts. We analyze the $\sim 580 \mathrm{~Hz}$ oscillations in the initial 0.75 seconds of the five bursts that were used in a previous search for a brightness oscillation at the expected $\sim 290 \mathrm{~Hz}$ spin frequency, and find that if the same frequency model describes all five bursts there is insufficient data to require more than a constant frequency or, possibly, a frequency plus a frequency derivative. Therefore, although it is appropriate to use an arbitrarily complicated model of the $\sim 580 \mathrm{~Hz}$ oscillations to generate a candidate waveform for the $\sim 290 \mathrm{~Hz}$ oscillations, models with more than two parameters are not required by the data. For the bursts as a whole we show that the characteristics of the brightness oscillations vary greatly from burst to burst. We find, however, that in at least one of the bursts, and possibly in three of the four that have strong brightness oscillations throughout the burst, the oscillation frequency reaches a maximum several seconds into the burst and then decreases. This behavior has not been reported previously for burst brightness oscillations, and it poses a challenge to the standard burning layer expansion explanation for the frequency changes.
\end{abstract}

Subject headings: X-rays: bursts — stars: neutron

\section{INTRODUCTION}

Shortly after the launch of the Rossi X-ray Timing Explorer (RXTE) in December 1995, observation with RXTE of neutron-star low-mass X-ray binaries (LMXBs) revealed that several sources had a single, highly coherent, high-amplitude brightness oscillation during at least one 
thermonuclear X-ray burst (for reviews see Strohmayer, Zhang, \& Swank 1997; Strohmayer, Swank, \& Zhang 1998a). The asymptotic frequency of these oscillations in the tails of bursts is so similar in different bursts from a single source, and the oscillation is so coherent in the tail (see, e.g., Strohmayer \& Markwardt 1999), that it is almost certain that this asymptotic frequency is the stellar spin frequency or its first overtone. These burst oscillations therefore provided the first direct evidence for the value of the spin frequencies of these LMXBs, and they corroborate strongly the proposed evolutionary link between LMXBs and millisecond rotation-powered pulsars. In addition, the stability of the frequency in the tails of the bursts has led to their application as promising probes of the binary systems themselves (Strohmayer et al. 1998b).

The existence of burst oscillations indicates that the emission from the surface, and hence the thermonuclear burning, is not uniform over the entire star. This is in accord with theoretical expectations (Joss 1978; Ruderman 1981; Shara 1982; Livio \& Bath 1982; Fryxell \& Woosley 1982; Nozakura, Ikeuchi, \& Fujimoto 1984; Bildsten 1995), and it suggests that the properties of the burst oscillations, such as the evolution of their frequency or amplitude, may contain valuable information about the propagation of thermonuclear burning over the surface of the neutron star. The lessons learned from study of the thermonuclear propagation in bursts may ultimately further our understanding of thermonuclear propagation in other astrophysical contexts, such as classical novae and Type Ia supernovae. Unlike in novae or Type Ia supernovae, burning in thermonuclear X-ray bursts occurs near the surface and occurs often for a single source, and is therefore relatively easy to observe. The detailed study of burst brightness oscillations therefore has broad importance.

Here we describe in detail the frequency behavior of the burst oscillations in five bursts from $4 \mathrm{U}$ 1636-536, which is an LMXB with an orbital period of 3.8 hours (see, e.g., van Paradijs et al. 1990). This source is of special interest because it produces detectable signals at both the fundamental and the first overtone of the stellar spin frequency (Miller 1999), and because near the beginning of one burst the brightness oscillations reached the highest amplitude $-50 \% \mathrm{rms}-$ so far recorded for oscillations during a thermonuclear burst (Strohmayer et al. 1998c).

In $\S 2$ we analyze the light curves of the bursts, and the frequency and amplitude of the brightness oscillations in the four of those five bursts that have strong brightness oscillations for most of the duration of the burst. We find that, despite apparent similarities in the light curves of three of those four bursts, the amplitude and frequency behavior of their brightness oscillations are very different from each other. We also find compelling evidence in one burst, and strong evidence in another burst, for an interval in which the burst oscillation frequency decreases after the peak in the light curve.

In $\S 3$ we focus on the initial portions of the bursts. Analyses of bursts from many sources have shown that the oscillation frequency often changes by a few Hertz over the first few seconds of a burst (see Strohmayer et al. 1998a for a review). The change is often a monotonic rise, but there are indications of more complicated behavior in some bursts. It has been pointed out that the 
Table 1: Starting Time of Bursts

\begin{tabular}{cc}
\hline \hline Burst & Date and Time \\
\hline a & $22: 39: 24.188$ UTC on 28 December 1996 \\
b & $23: 54: 02.876$ UTC on 28 December 1996 \\
c & $23: 26: 46.813$ UTC on 29 December 1996 \\
d & $17: 36: 52.941$ UTC on 31 December 1996 \\
e & 09:57:25.938 UTC on 23 February 1997 \\
\hline
\end{tabular}

magnitude of the frequency change could be explained by a 20-50 meter expansion of the burning layers followed by a slow settling, if the layers conserve angular momentum (see, e.g., Strohmayer et al. 1998a), but details have not been worked out. For example, it is not clear how the layers would maintain their coherence throughout the 5-10 complete circuits relative to the body of the star that are implied by the observations. Bildsten (1998) has suggested that the layers may be stabilized by thermal buoyancy or mean molecular weight stratification, but details have not been worked out. In $\S 3$ we examine in detail the first 0.75 seconds of all five bursts, which was the interval used to construct the candidate waveform for the $\sim 290 \mathrm{~Hz}$ oscillation in $4 \mathrm{U} 1636-536$ (Miller 1999). We examine models of the frequency behavior that have increasing complexity: a constant-frequency model; one with a frequency and frequency derivative; a four-parameter model with an initial frequency and frequency derivative followed by a different frequency derivative after a break time; and a five-parameter model with two different frequencies and frequency derivatives separated by a break time. We find that if the same type of frequency model applies to all five of the bursts then the data do not require a model more complicated than the constant-frequency model or, possibly, the model with a single frequency and frequency derivative. Note, however, that this is not inconsistent with the use of the five-parameter model to construct a waveform used in the search for the expected $\sim 290 \mathrm{~Hz}$ oscillation (Miller 1999); in such a search, the only goal is to find the best fit to the $\sim 580 \mathrm{~Hz}$ oscillations, and the extra parameters need not be justified by a significantly better fit.

Finally, in $\S 4$ we discuss the implications of these results for the current picture of the frequency changes, in which the frequency change occurs because the burning layer is lifted by 20-50 meters from the surface by the radiation flux. We find that the simplest version of this picture has difficulty explaining the observations.

\section{OVERVIEW OF THE BURSTS}

We used public-domain data from the High Energy Astrophysics Science Archive Research Center. The data were taken in Single Bit Mode, which does not record the energy of photons. We give the starting times of the bursts in Table 1 and the light curves in Figure 1. In burst d, the data dropouts are caused by telemetry saturation. 


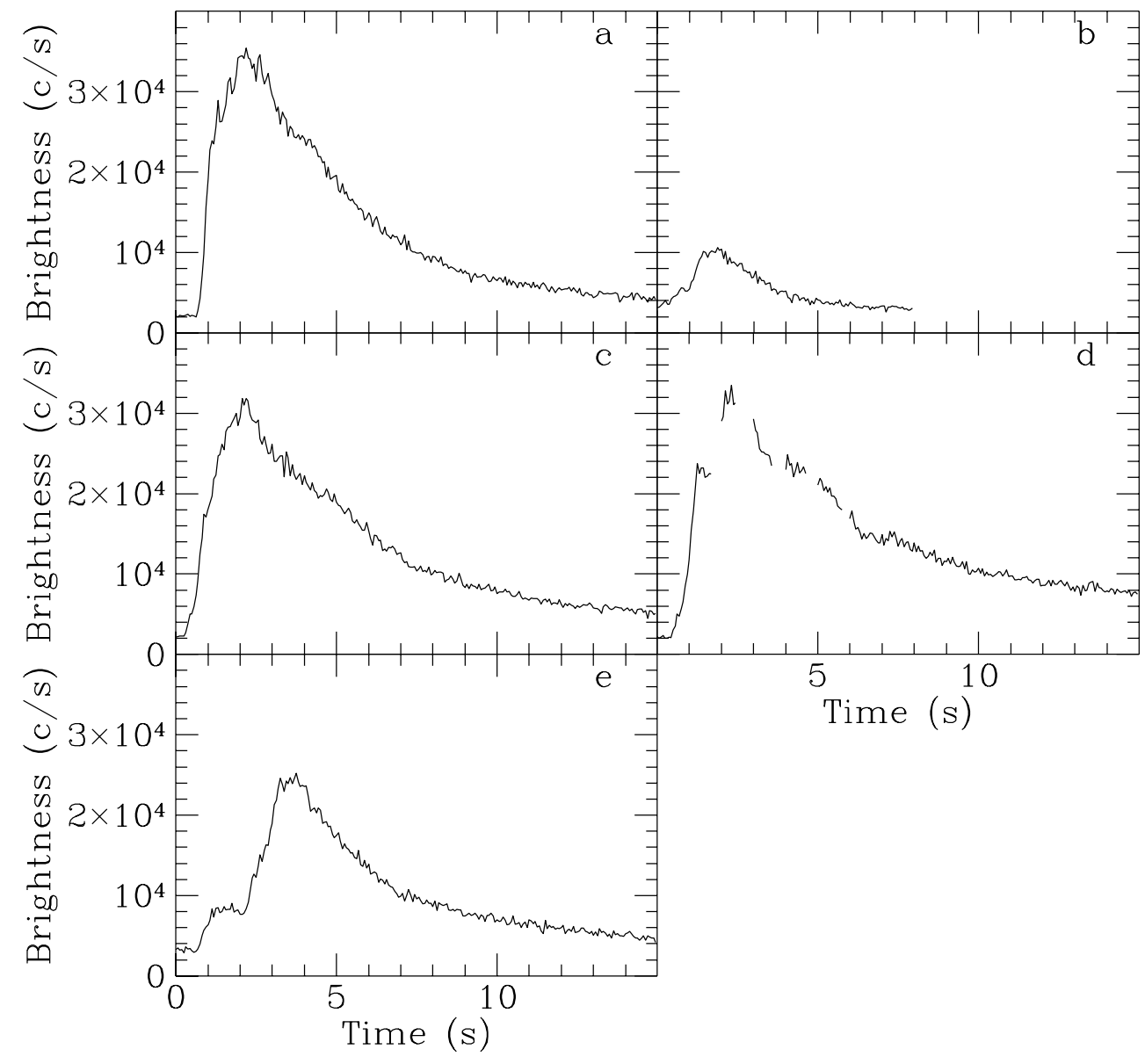

Fig. 1. - Light curves for the bursts. (a) Burst beginning at 22:39:24 UTC on 28 December 1996. (b) Burst beginning at 23:54:02 UTC on 28 December 1996. (c) Burst beginning at 23:26:46 UTC on 29 December 1996. (d) Burst beginning at 17:36:52 UTC on 31 December 1996. (e) Burst beginning at 09:57:26 UTC on 23 February 1998. The data gaps in burst d are caused by telemetry saturation. 
Figure 2 shows the peaks of the power spectra of the first four bursts, as a function of time. The burst on 23 February 1997 does not have a strong brightness oscillation for most of its duration, and we therefore do not analyze it in the rest of this section. For each burst, the frequency of maximum power in successive nonoverlapping one-second intervals is shown by the solid triangles, and the Leahy et al. (1983)-normalized power at this frequency is shown by the solid line. Here we plot only those points with Leahy powers in excess of 10 (chance probability for a single trial less than $6 \times 10^{-3}$ ). The horizontal bars on the frequency points indicate the extent of the interval for which the power density spectrum was calculated. In a few cases, more than one peak exceeds this threshold in a given power density spectrum. We then represent the lower-power peak by an open circle. In burst a the secondary peak has a Leahy power of 21.2 (single-trial significance $2.5 \times 10^{-5}$ ); in burst b the secondary peaks have Leahy powers of 44.0 (first interval; significance $2.8 \times 10^{-10}$ ) and 13.5 (second interval; significance $1.2 \times 10^{-3}$ ); and in burst $\mathrm{c}$ the secondary peak has a Leahy power of 12.8 (significance $1.7 \times 10^{-3}$ ). Finally, Figure 3 shows the rms amplitude of each oscillation, computed for one-second intervals $1 / 8$ second apart.

It is evident from these figures that the frequency behavior can be very complex and can differ greatly from burst to burst. The light curves for bursts (a), (c), and (d) appear similar to each other, although burst (d) has a slightly longer decay time than the other two. However, the frequency and amplitude of the brightness oscillations evolve very differently in the three bursts.

In burst (a), there is a strong oscillation near the beginning which disappears for approximately one second, then the oscillation reappears after the peak. The frequency increases continuously, although there is some evidence that in the initial $\sim 0.5$ second of the burst the frequency drops (this might help explain the presence of a higher-frequency secondary peak in the power density spectrum).

In burst (c), the brightness oscillation is present for almost the entire time examined. The frequency increases rapidly in the first two to three seconds, then appears to decrease to an asymptotic value. A power density spectrum of a two-second interval starting 1.75 seconds after the beginning of the burst reveals a peak at $581.62 \pm 0.04 \mathrm{~Hz}$. A power density spectrum of a six-second interval starting four seconds after the beginning of the burst has a peak at $581.47 \pm 0.01 \mathrm{~Hz}$. If the latter frequency is the asymptotic frequency of the oscillation, then at a $3 \sigma$ level of certainty it is less than the maximum frequency attained during the burst. The amplitude of the oscillation in the burst tail is high and significant, and there is an abrupt increase in the amplitude 6-8 seconds after the beginning of the burst that is not accompanied by any apparent change in the light curve.

In burst (d) there is a clear decrease in the frequency of the burst oscillation in the tail of the burst. We explored this further by taking a power density spectrum of a longer interval: five seconds, starting three seconds after the beginning of the burst. We found that, at the $99.99 \%$ confidence level, the frequency change per second during this interval is $-0.54 \pm 0.08 \mathrm{~Hz} \mathrm{~s}{ }^{-1}$. The best-fit frequency at the beginning of this five-second interval depends on the frequency derivative, 


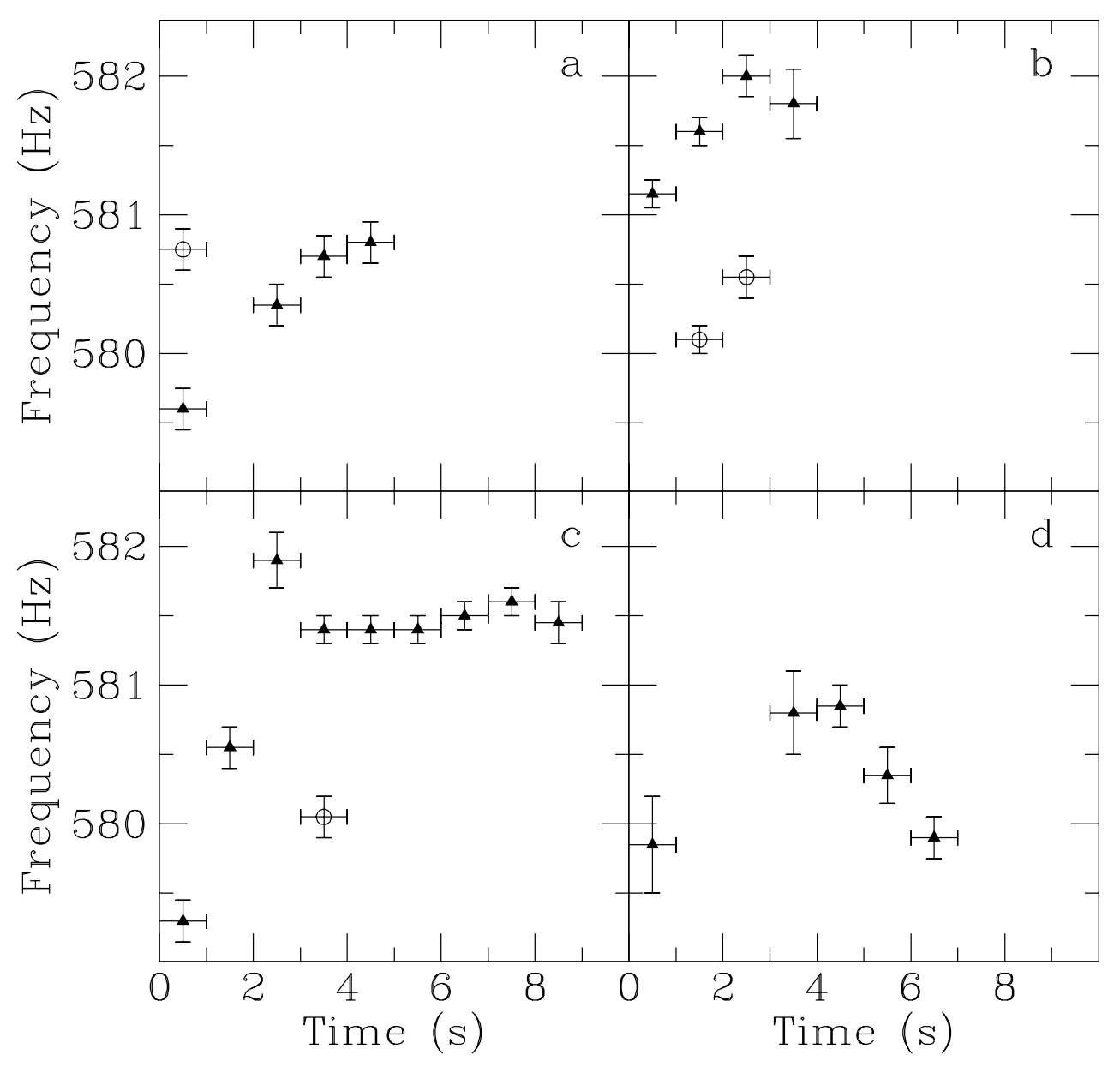

Fig. 2.- Power spectra as a function of time for the four bursts with strong brightness oscillations. Each solid triangle is at the frequency of maximum power and its $1 \sigma$ uncertainties for nonoverlapping 1 second intervals. The frequency of a peak is only plotted if its Leahy power exceeds 10. In bursts 1, 2, and 3 there are intervals in which a second peak exceeds this threshold, and this secondary peak is plotted with an open circle. The power of the secondary peak in burst (a) is 21.2 (single-trial significance $2.5 \times 10^{-5}$ ); the powers of the secondary peaks in burst (b) are 44.0 (significance $2.8 \times 10^{-10}$ ) and 13.5 (significance $1.2 \times 10^{-3}$ ); and the power of the peak in burst (c) is 12.8 (significance $1.7 \times 10^{-3}$ ). The panels are labeled as in Figure 1. Burst (e) does not have strong brightness oscillations for most of the burst, and is therefore excluded from this analysis. 


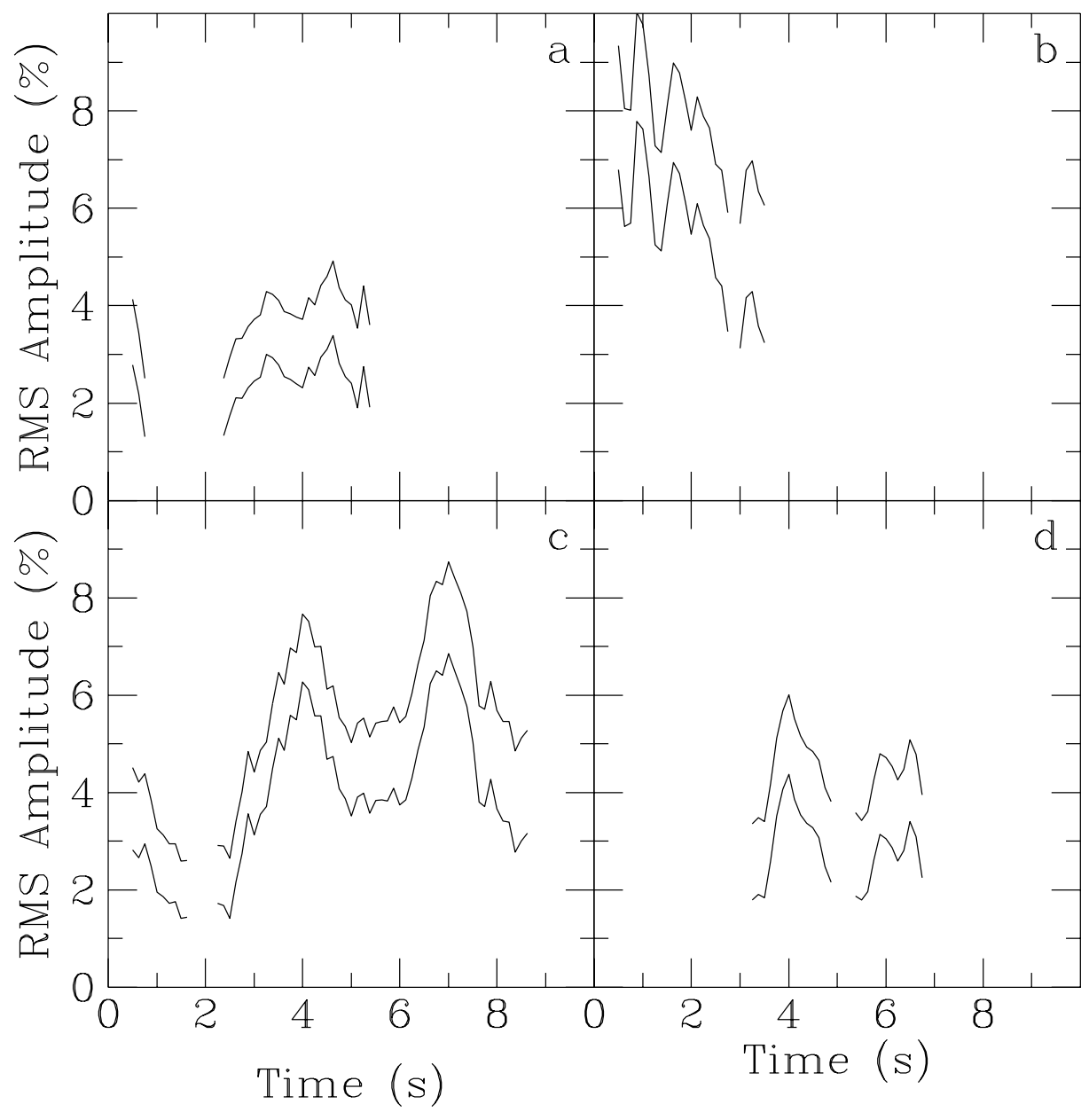

Fig. 3.- Root mean square amplitude of the brightness oscillation for each of the bursts. The amplitudes are calculated for one-second intervals with starting times $1 / 8$ second apart, and the $\pm 1 \sigma$ uncertainty bands are shown. The amplitude is only plotted if the Leahy power for the oscillation exceeds 10. The panels are labeled as in Figure 1. 
and is approximately $\nu_{0}=581.39 \mathrm{~Hz}-2\left(\dot{\nu}+0.62 \mathrm{~Hz} \mathrm{~s}^{-1}\right) \mathrm{Hz}$. This means that, relative to a brightness oscillation with a constant frequency equal to the frequency at the beginning of this five-second interval, the observed brightness oscillation has a total phase lag of between $12 \pi$ and $16 \pi$ radians. The total phase lag is comparable to what is seen in many bursts, except that here the frequency inferred in the tail of the burst is significantly less than the spin frequency inferred from other bursts in this source. There is no sign in this burst that the frequency has reached an asymptotic value.

Burst (b) is the only one with a clearly different light curve. This is a weak burst. The frequency of the brightness oscillation is consistent with what is observed in, at least, bursts (a) and (c): a rise in the frequency near the beginning of the burst, followed by an approximate leveling off. We note, however, that within the uncertainties the frequency could also reach a maximum and then decline, as appears to be the case for bursts (c) and (d).

\section{BRIGHTNESS OSCILLATIONS AT THE BEGINNING OF THE BURST}

Previous analyses have shown that the brightness oscillations in the initial $\sim$ second of the bursts are often of particular interest. This is where the highest amplitudes ( $\mathrm{rms} \sim 50 \%$; Strohmayer et al. 1998c) are reported, and where subharmonics of the strong oscillation have been detected in 4U 1636-536 (Miller 1999) and possibly in the Rapid Burster (Fox \& Lewin 1999). It is therefore important to examine the initial portion more closely to see what hints about the brightness oscillation mechanism can be derived.

Before doing so, we need to emphasize an important distinction. If the purpose of the analysis is to characterize the frequency variations of the $\sim 580 \mathrm{~Hz}$ oscillation then extra parameters can only be added if the fit to the data is improved sufficiently to justify the additional complexity. The situation is different when the goal is to produce a matched filter for a search for a harmonically related frequency, as in the search for a signal at half of the $\sim 580 \mathrm{~Hz}$ dominant brightness oscillation in 4U 1636-536 (Miller 1999). For that purpose, it is not necessary to justify the extra parameters used in the construction of the filter, if no reference is made to the signal for which one is searching. In the case of the search for the $\sim 290 \mathrm{~Hz}$ oscillation, a five-parameter matched filter was used for each burst; matched filters with fewer parameters also give a clear signal at $\sim 290 \mathrm{~Hz}$, although with lower significance because the filter does not fit the data as well.

A general method to find the best-fit values of parameters and their confidence regions employs a likelihood function. In this approach, we suppose that we have a model in which the countrate as a function of time is predicted to be $s(t)$, from which we can predict the number of counts $s_{i}$ in one particular bin $i$ of the data, which in this case is $1 / 8192 \mathrm{~s}$ in duration. In general, $s_{i}$ is not an integer. The actual number of counts observed in bin $i$ is $c_{i}$, which is an integer. With 
these definitions, the Poisson likelihood of the full data set given the model $s(t)$ is

$$
\mathcal{L}=\Pi \frac{s_{i}^{c_{i}}}{c_{i} !} e^{-s_{i}}
$$

where the product is over all of the bins of the data. Note that in normal applications of the point likelihood the bin sizes would be so small that a given bin would have either zero or one count, but the fixed bin size of $1 / 8192 \mathrm{~s}$ combined with the high count rates during the bursts (up to $\sim 30,000 \mathrm{c} / \mathrm{s}$; see Figure (1) means that many of the bins have multiple counts. The likelihood is maximized to determine the best values of the parameters of the model waveform $s(t)$, and approximate confidence contours can be estimated using contours of constant log likelihood: $2 \Delta \log \mathcal{L}=\Delta \chi^{2}$ (Eadie et al. 1971, $\S 9.4 .3$, p. 207).

The model waveform $s(t)$ will in general include components related to the relatively slow change in the brightness of the source as well as components related to the high-frequency brightness oscillation. However, the frequency scales are different enough $(\sim 1-5 \mathrm{~Hz}$ for the slowly rising component versus $\sim 580 \mathrm{~Hz}$ for the fast oscillations) that the fitting of the two components are nearly independent of each other. This means that, when we analyze the behavior of the brightness oscillations, we can simplify by assuming that the burst has a constant average brightness. With this in mind, the model we consider is

$$
s(t)=c_{\mathrm{av}}\left(1+A \cos 2 \pi\left[\nu(t) t+\phi_{0}\right]\right),
$$

where $c_{\mathrm{av}}$ is the average countrate, $A$ is the amplitude of the signal (which we assume to be time-independent), $\nu(t)$ is the frequency as a function of time, and $\phi_{0}$ is the phase of the oscillation at the beginning of the data interval analyzed.

In this section we explore four different models for the frequency behavior in the initial 0.75 seconds of each of the four bursts (this time was chosen to conform to the analysis of Miller [1999], which was performed to look for a weaker $\sim 290 \mathrm{~Hz}$ oscillation). The four models are:

$$
\begin{gathered}
\nu_{1}(t)=\nu_{0} \\
\nu_{2}(t)=\nu_{0}+\dot{\nu} t \\
\nu_{3}(t)=\nu_{1}+\dot{\nu}_{1} t, \quad t<t_{\text {break }} \\
=\nu_{2}+\dot{\nu}_{2} t, \quad t>t_{\text {break }}
\end{gathered}
$$

where continuity of the frequency is imposed, so that there are four independent parameters, and finally

$$
\begin{aligned}
\nu_{4}(t) & =\nu_{1}+\dot{\nu}_{1} t, \quad t<t_{\text {break }} \\
& =\nu_{2}+\dot{\nu}_{2} t, \quad t>t_{\text {break }}
\end{aligned}
$$

where continuity of the frequency is not imposed, so that there are five independent parameters.

For the purposes of this section the most interesting of the parameters of the model waveform $s(t)$ is the frequency, as opposed to the amplitude or the initial phase of the brightness oscillation. 
If the amplitude $A \ll 1$, as it is in this case, then a tremendous speed-up in the search procedure is possible with the use of the cross-correlation (see, e.g., Helstrom 1960 or Wainstein \& Zubakov 1962 for details of cross-correlation and matched filtering techniques)

$$
H=C\left|\int_{t_{0}}^{t_{0}+T} c(t) e^{-i \nu(t) t} d t\right|^{2},
$$

where $t_{0}$ is the start time of the burst, $T=0.75 \mathrm{~s}$ is the duration of the burst, and $C$ is a normalization constant. In practice this integral is actually calculated as a sum over all of the bins of the data, and $d t=1 / 8192 \mathrm{~s}$ is the duration of a bin. If $C=2 / N_{\text {tot }}$, where $N_{\text {tot }}$ is the total number of counts in the data set, then $H$ has the same statistical properties as the Leahy power; $H$ is also related to the $Z^{2}$ statistic used in pulsar period searches (Buccheri et al. 1983; see Strohmayer \& Markwardt 1999 for a recent use in the characterization of brightness oscillations during thermonuclear X-ray bursts). To lowest order in the oscillation amplitude $A$ this description is mathematically identical to the likelihood description, but it is much faster to apply because no search need be performed for the amplitude or oscillation phase. It is therefore preferable for low-amplitude oscillations.

With this formalism, we can estimate the best values and uncertainty regions for the different frequency models above. The figures in the previous section, which were constructed using a constant-frequency waveform, give this information for the one-parameter, constant-frequency model.

\subsection{Two-Parameter Frequency Model}

The best-fit values for the two-parameter frequency model are given in Table 2. To estimate uncertainties on these parameters, we performed a Monte Carlo analysis in which we selected $10^{6}$ random values per burst of $\nu_{1}, \dot{\nu}_{1}, \dot{\nu}_{2}$, and $t_{\text {break }}$, uniformly sampled from, respectively, $576 \mathrm{~Hz}$ to $585 \mathrm{~Hz} ;-12 \mathrm{~Hz} \mathrm{~s}^{-1}$ to $12 \mathrm{~Hz} \mathrm{~s}^{-1} ;-12 \mathrm{~Hz} \mathrm{~s}^{-1}$ to $12 \mathrm{~Hz} \mathrm{~s}^{-1}$; and $0 \mathrm{~s}$ to $0.75 \mathrm{~s}$. The quoted uncertainties for single parameters were computed using a Bayesian viewpoint, in which the posterior probability density was calculated throughout the interval and then integrated over the other three parameters to produce a marginalized probability distribution. We have assumed a uniform prior probability density over the whole space searched. This means that the posterior probability density is simply proportional to the likelihood. These confidence regions, which are the smallest regions that encompass $68 \%$ of the probability, are given in Table 3 . In some cases the maximum likelihood value of a parameter obtained by extremization in the full two-dimensional parameter space is outside the marginalized $68 \%$ confidence region. This is symptomatic of the fact that the parameters are constrained only weakly by the data. 
Table 2: Best-Fit Parameters for Two-Parameter Model

\begin{tabular}{ccc}
\hline \hline Burst & $\nu_{1}(\mathrm{~Hz})$ & $\dot{\nu}_{1}\left(\mathrm{~Hz} \mathrm{~s}^{-1}\right)$ \\
\hline $\mathrm{a}$ & 579.0 & 4.0 \\
$\mathrm{~b}$ & 580.3 & 2.0 \\
$\mathrm{c}$ & 581.0 & -3.8 \\
$\mathrm{~d}$ & 578.6 & 4.2 \\
$\mathrm{e}$ & 581.1 & -3.4 \\
\hline
\end{tabular}

Table 3: $68 \%$ Confidence Regions for Two-Parameter Model

\begin{tabular}{ccc}
\hline \hline Burst & $\nu_{1}(\mathrm{~Hz})$ & $\dot{\nu}_{1}\left(\mathrm{~Hz} \mathrm{~s}^{-1}\right)$ \\
\hline $\mathrm{a}$ & $579.7-580.1$ & $-8.0-8.0$ \\
$\mathrm{~b}$ & $580.9-581.2$ & $-8.8-8.0$ \\
$\mathrm{c}$ & $579.2-579.7$ & $-8.0-8.0$ \\
$\mathrm{~d}$ & $578.8-579.9$ & $-8.0-8.0$ \\
$\mathrm{e}$ & $579.2-583.6$ & $-8.0-8.0$ \\
\hline
\end{tabular}

Table 4: Best-Fit Parameters for Four-Parameter Model

\begin{tabular}{ccccc}
\hline \hline Burst & $\nu_{1}(\mathrm{~Hz})$ & $\dot{\nu}_{1}\left(\mathrm{~Hz} \mathrm{~s}^{-1}\right)$ & $\dot{\nu}_{2}\left(\mathrm{~Hz} \mathrm{~s}^{-1}\right)$ & $t_{\text {break }}(\mathrm{s})$ \\
\hline $\mathrm{a}$ & 581.3 & -9.0 & 11.2 & 0.28 \\
$\mathrm{~b}$ & 579.9 & 4.0 & 0.5 & 0.32 \\
$\mathrm{c}$ & 579.1 & 8.7 & -6.7 & 0.20 \\
$\mathrm{~d}$ & 579.7 & -2.4 & 7.9 & 0.28 \\
$\mathrm{e}$ & 583.2 & -12.0 & -0.8 & 0.28 \\
\hline
\end{tabular}

\subsection{Four-Parameter Frequency Model}

The best-fit values for the four-parameter frequency model are given in Table 4. As for the two-parameter model, the uncertainties were estimated by marginalizing over all but the parameter of interest; the confidence regions containing $68 \%$ of the probability are given in Table 5 .

\subsection{Five-Parameter Frequency Model}

The best-fit values for the five-parameter frequency model are given in Table 6 . As for the two-parameter model, the uncertainties were estimated by marginalizing over all but the parameter of interest; the confidence regions containing $68 \%$ of the probability are given in Table 7 . 
Table 5: 68\% Confidence Regions for Four-Parameter Model

\begin{tabular}{ccccc}
\hline \hline Burst & $\nu_{1}(\mathrm{~Hz})$ & $\dot{\nu}_{1}\left(\mathrm{~Hz} \mathrm{~s}^{-1}\right)$ & $\dot{\nu}_{2}\left(\mathrm{~Hz} \mathrm{~s}^{-1}\right)$ & $t_{\text {break }}(\mathrm{s})$ \\
\hline $\mathrm{a}$ & $579.0-581.0$ & $-8.8-2.4$ & $2.4-6.4$ & $0.16-0.42$ \\
$\mathrm{~b}$ & $578.8-580.6$ & $-0.8-8.0$ & $-4.0-3.2$ & $0.13-0.63$ \\
$\mathrm{c}$ & $579.0-581.6$ & $-7.2-5.6$ & $-5.6-4.0$ & $0.13-0.64$ \\
$\mathrm{~d}$ & $578.0-579.8$ & $-4.8-5.6$ & $-4.0-6.4$ & $0.11-0.64$ \\
$\mathrm{e}$ & $579.2-582.0$ & $-8.0-4.0$ & $-5.6-4.8$ & $0.14-0.63$ \\
\hline
\end{tabular}

Table 6: Best-Fit Parameters for Five-Parameter Model

\begin{tabular}{cccccc}
\hline \hline Burst & $\nu_{1}(\mathrm{~Hz})$ & $\nu_{2}(\mathrm{~Hz})$ & $\dot{\nu}_{1}\left(\mathrm{~Hz} \mathrm{~s}^{-1}\right)$ & $\dot{\nu}_{2}\left(\mathrm{~Hz} \mathrm{~s}^{-1}\right)$ & $\mathrm{t}_{\text {break }}(\mathrm{s})$ \\
\hline $\mathrm{a}$ & 581.2 & 578.0 & -7.6 & 6.4 & 0.34 \\
$\mathrm{~b}$ & 579.0 & 579.6 & 8.0 & 3.2 & 0.34 \\
$\mathrm{c}$ & 578.6 & 578.0 & 6.8 & 0.8 & 0.41 \\
$\mathrm{~d}$ & 578.0 & 582.6 & -0.4 & -5.2 & 0.25 \\
$\mathrm{e}$ & 582.0 & 585.0 & -8.0 & -0.4 & 0.41 \\
\hline
\end{tabular}

Table 7: 68\% Confidence Regions for Five-Parameter Model

\begin{tabular}{cccccc}
\hline \hline Burst & $\nu_{1}(\mathrm{~Hz})$ & $\nu_{2}(\mathrm{~Hz})$ & $\dot{\nu}_{1}\left(\mathrm{~Hz} \mathrm{~s}^{-1}\right)$ & $\dot{\nu}_{2}\left(\mathrm{~Hz} \mathrm{~s}^{-1}\right)$ & $\mathrm{t}_{\text {break }}(\mathrm{s})$ \\
\hline $\mathrm{a}$ & $579.6-581.8$ & $577.4-581.6$ & $-8.8-4.8$ & $0.0-6.4$ & $0.11-0.48$ \\
$\mathrm{~b}$ & $577.8-580.6$ & $579.4-582.0$ & $-5.6-8.8$ & $-2.4-3.2$ & $0.08-0.52$ \\
$\mathrm{c}$ & $577.8-581.2$ & $577.4-581.8$ & $-6.4-8.8$ & $-5.6-1.6$ & $0.09-0.63$ \\
$\mathrm{~d}$ & $579.2-581.6$ & $577.0-581.6$ & $-9.6-6.4$ & $-2.4-6.4$ & $0.08-0.34$ \\
$\mathrm{e}$ & $579.0-582.8$ & $577.6-582.4$ & $-8.8-6.4$ & $-4.8-3.2$ & $0.08-0.56$ \\
\hline
\end{tabular}

\subsection{Summary of Frequency Models}

The best-fit parameters and relative log likelihoods are listed in Table 8; as indicated above, $2 \Delta \log \mathcal{L} \approx \Delta \chi^{2}$. From this table, it is clear that for all but burst four it is not necessary to use the five-parameter fit, and for bursts 2,3 , and 4 it is not necessary to use a model more complicated than the two-parameter model in which the frequency and frequency derivative are constant throughout the first 0.75 seconds. For burst (d) by itself the five-parameter model is preferred at only the $2 \sigma$ level compared to the four-parameter model, and for all five bursts combined the five-parameter model is preferred at less than the $1 \sigma$ level relative to the four-parameter model. For all five bursts combined, the four-parameter model is preferred at less than the $1 \sigma$ level compared to the two-parameter model, and the two-parameter model is preferred at less than the $2 \sigma$ level compared to the one-parameter model. 
Table 8: Relative Log Likelihoods for Different Models

\begin{tabular}{ccccc}
\hline \hline Burst & 1-Param & 2-Param & 4-Param & 5-Param \\
\hline $\mathrm{a}$ & 0.0 & 1.3 & 4.3 & 4.5 \\
$\mathrm{~b}$ & 0.0 & 1.2 & 1.6 & 2.8 \\
$\mathrm{c}$ & 0.0 & 1.3 & 2.0 & 3.1 \\
$\mathrm{~d}$ & 0.0 & 2.2 & 3.2 & 5.4 \\
$\mathrm{e}$ & 0.0 & 0.6 & 1.0 & 2.0 \\
\hline
\end{tabular}

\section{DISCUSSION AND SUMMARY}

What can be learned from this detailed characterization of the burst brightness oscillations in $4 \mathrm{U} 1636-536$ ? The clearest impression left is that there are no simple statements about the frequency behavior that are true for all of the bursts. In two of the bursts, one can make an argument that the oscillation frequency is initially $1-2 \mathrm{~Hz}$ below the asymptotic frequency, and then rises. In this interpretation, the asymptotic frequency is extremely close to the spin frequency of the neutron star. This picture can be qualitatively explained by the idea that the burning layer lifts 20-50 meters during the burst and settles down gradually. However, the burst on 31 December 1996 does not follow this pattern. The frequency in the initial second is indeed lower than the maximum value attained, but the significance of this initial signal is low (Leahy power of 10). The maximum is followed by a clear decrease in the frequency over several seconds, with a total phase change equivalent to more than five complete circuits around the star. This happens during a time when the countrate decreases from approximately $2 / 3$ of the maximum to approximately $1 / 3$ of the maximum. The burst on 29 December 1996 has a very strong and significant brightness oscillation in its tail, which appears to level out to a constant frequency. However, near the peak of the light curve for this burst the oscillation frequency is higher than this asymptotic frequency, at a $3 \sigma$ significance level.

Such a drop in frequency is not expected in the simplest version of the hypothesis that the frequency changes are caused by the rise of the burning layers. In this model, the highest frequency should be observed when the layers are fully coupled to the core of the star, which is expected to occur when the frequency has reached its asymptotic limit.

Another constraint on the hypothesis that the asymptotic frequency equals the spin frequency (after correcting for orbital Doppler shifts) is that the variation in the observed asymptotic frequency must be consistent with the possible modulation due to the binary motion of the neutron star. From binary evolution theory (see, e.g., Lamb \& Melia 1987; Verbunt \& van den Heuvel 1995), an LMXB such as $4 \mathrm{U}$ 1636-536 with a $3.8 \mathrm{hr}$ orbital period (van Paradijs et al. 1990) that contains a neutron star of mass $M_{\mathrm{NS}}=1.4 M_{\odot}$ to $2.0 M_{\odot}$ has a companion star of mass $M_{c} \approx 0.4 M_{\odot}$. Assuming that the orbit is approximately circular, the orbital velocity of the neutron star is therefore $90-130 \mathrm{~km} \mathrm{~s}^{-1}$, implying a maximum frequency modulation of $\Delta \nu / \nu=4.3 \times 10^{-4}$, or approximately $0.25 \mathrm{~Hz}$ if $\nu=580 \mathrm{~Hz}$. Therefore, the observed asymptotic frequency cannot be 
different by more than $0.5 \mathrm{~Hz}$ for two different bursts. The analysis of the 31 December 1996 burst reported in $\S 2$ indicates that eight seconds after the start of the burst the frequency is less than $579.0 \mathrm{~Hz}$. The asymptotic frequency in the burst on 29 December 1996 is $581.43 \mathrm{~Hz}$, so the frequency in the 31 December 1996 burst must rise by $2 \mathrm{~Hz}$ to reach a plausible spin frequency.

It is difficult to reconcile this frequency behavior with what is expected in the simplest version of the rising burning layer hypothesis. One possibility is that the observed frequency changes are not simply indicative of the spin frequency of the burning layer, but also include a time-dependent change in the phase at which the photons emerge relative to the phase of the burning layer. This would be observationally indistinguishable from a pure frequency change, and would add an extra degree of freedom to the model.

Even this, however, is subject to significant observational restrictions. To see this, consider the following observational trends, which have been observed in many bursts from several sources (see, e.g., Strohmayer et al. 1998 for a summary). In the remainder of this section we assume that all quantities (e.g., frequencies, times, and phases) are measured at infinity.

(1) There are several bursts in which burst oscillations are seen for the entire burst, and do not disappear during the time of peak countrate.

(2) Aside from an early phase in which there may be a frequency decrease, the frequency increases smoothly as the burst progresses.

(3) The total phase lag of the oscillations compared with a hypothetical oscillation that has a constant frequency equal to the frequency in the burst tail is as much as $10 \pi$.

The total amount of energy in a burst is $\sim 10^{39}$ ergs. If expansion of a layer and angular momentum conservation are to explain the $\sim 0.3 \%-1 \%$ change in the observed angular frequency, then the layer must rise by a distance that is a fraction $\sim 0.2 \%-0.5 \%$ of the radius of the star, or 20 to 50 meters. The surface gravity of a neutron star is $\sim 2 \times 10^{14} \mathrm{~cm} \mathrm{~s}^{-2}$, so the largest amount of mass that can be lifted to the required $20-50$ meter height above the surface is $\sim 1-2 \times 10^{21} \mathrm{~g}$. If most of the $\sim 10^{13} \mathrm{~cm}^{2}$ surface area of the star is involved, this implies that the greatest column depth which could be lifted to the required height is roughly $10^{8} \mathrm{~g} \mathrm{~cm}^{-2}$, which is comparable to the expected $10^{6}-10^{8} \mathrm{~g} \mathrm{~cm}^{-2}$ depth of ignition (see, e.g., Fushiki \& Lamb 1987; Brown \& Bildsten 1998).

One may therefore distinguish two scenarios: (1) the burning layer rotates with the core of the star at a constant spin frequency and the observed frequency shifts are caused by phase shifts induced by radiation transport through more slowly rotating layers, and (2) the burning layer itself is lifted and rotates more slowly than the core of the star. We now treat these in order.

Suppose for simplicity that the burning layer has an infinitesimal vertical extent, that it has some restricted azimuthal extent, and that it all rotates with the same angular frequency $\omega_{\text {burn }}(t)$. The energy from this layer propagates upwards through the atmosphere, which in general may be composed of layers with different angular frequencies. Therefore, the phase of emergence of 
the radiation may differ from the phase of the burning layer at the time of the emission of the radiation. Under the rising burning layer hypothesis, it is expected that the angular frequency of higher layers is less than the angular frequency of lower layers $(d \omega / d h<0)$. Hence, there is expected to be a lag $\phi_{\text {lag }}>0$ between the phase of emergence and the phase of emission. This phase lag will, in general, have a time-dependence, as the scale height of the atmosphere and the angular frequency of different layers in the atmosphere changes throughout the burst. An observer at infinity will therefore see a net angular frequency of a hot spot that is equal to $\omega_{\text {burn }}(t)-\dot{\phi}_{\operatorname{lag}}(t)$.

Consider first a burning layer that rotates with the stellar core throughout the burst. Then $\omega_{\text {burn }}(t)=$ const $=\omega_{\text {spin }}$. If neither $\omega(h)$ nor the density or height of the envelope changes with time, then $\phi_{\text {lag }}$ is a constant and the observed frequency is just $\omega_{\text {spin }}$. Hence, in order to have an apparent frequency shift in this situation, the structure or angular velocity of the envelope must change with time.

Now consider an envelope that does change with time. For us to observe a frequency less than $\omega_{\text {spin }}$, it is necessary that $\dot{\phi}_{\text {lag }}(t)>0$, so the characteristic phase of emergence of the radiation must lag the phase of the source of heat by a greater and greater amount with increasing time (the increase of this phase lag with time must itself decrease with time to produce the observed increase in frequency). But how is this possible? As the envelope settles down, the phase lag should decrease, because $d \omega(t) / d h<0$. But if the phase lag decreases, the observed frequency should be higher than the spin frequency. This is not seen in most bursts, and even in the burst on 29 December 1996 where there does appear to be a short period of spindown, the total phase lead implied by the spindown is much smaller than the total phase lag implied by the spinup near the beginning of the burst. Thus, the preceding set of assumptions is inconsistent with the data.

This demonstrates that the observed frequency behavior is inconsistent with the source of heat (i.e., the burning layer) rotating at a constant frequency equal to $\omega_{\text {spin }}$. Instead, the source of heat must change its frequency during the burst.

To analyze this situation, let us now consider a burning layer with a finite thickness, so that the observed photons are a superposition of the photons from many infinitesimal layers such as discussed above. The observed frequency of oscillation is then a superposition of the frequencies due to the infinitesimal layers.

Consider two of these infinitesimal slices, labeled 1 and 2 , where slice 1 is higher than slice 2. Suppose that these slices are not coupled to each other. Then, by assumption, the angular frequency $\omega_{\text {burn,1 }}$ of slice 1 is less than the angular frequency $\omega_{\text {burn,2 }}$ of slice 2 . In addition, because the photons from slice 2 have to travel through the same atmospheric layers as the photons from slice 1 in addition to the layers between 2 and 1, the phase lag $\phi_{\text {lag, }, 1}$ of photons from slice 1 is expected to be less than the phase lag $\phi_{\mathrm{lag}, 2}$ of photons from slice 2 . Hence, as the atmospheric scale height decreases, it is expected that $\phi_{\operatorname{lag}, 2}$ will decrease more rapidly than $\phi_{\text {lag, } 1}$ does, so that

$$
\dot{\phi}_{\operatorname{lag}, 2}<\dot{\phi}_{\operatorname{lag}, 1}<0
$$


Therefore, the difference between the angular frequency of the photons from slice 2 and the angular frequency of the photons from slice 1 is

$$
\omega_{\mathrm{burn}, 2}-\omega_{\mathrm{burn}, 1}+\dot{\phi}_{\mathrm{lag}, 1}-\dot{\phi}_{\mathrm{lag}, 2}>\omega_{\mathrm{burn}, 2}-\omega_{\mathrm{burn}, 1} .
$$

This means that the phases of emergence of radiation diverge rapidly from each other, which leads quickly to a low amplitude unless the heat source has a small vertical extent. The requirement that the amplitude be significant means that the total azimuthal phase subtended by the emergent radiation has to be much less than $2 \pi$. The integrated phase lag relative to the stellar core is often $10 \pi$ or larger, hence the average vertical extent of the heat source must be much less than $1 / 5$ of the vertical distance from the original location of the heat source to its location during the burst. An alternative to having the vertical extent of the layer be small is that the burning layer may be tightly coupled to itself, so that its angular frequency is approximately constant over a significant vertical distance.

To summarize, several conclusions may be drawn about the standard model for frequency changes during burst oscillations, which we take to be the picture that at least part of the burning layer is lifted and then settles gradually to the surface as the flux drops, producing an observed asymptotic frequency equal to the spin frequency of the neutron star Doppler-shifted by the orbital motion of the neutron star. (1) The burning region itself (and not just overlaying optically thick layers) must be lifted by 20-50 meters from the surface, (2) this region must remain decoupled from the rest of the star, presumed to be rotating at the original spin frequency, for several seconds, (3) to produce the observed coherence of the brightness oscillations during the rise in frequency, the burning layer must either have a vertical extent much smaller than its height above the surface or be strongly coupled to itself to prevent relative azimuthal motion, and (4) the existence of a frequency greater than the asymptotic frequency (as in the 29 December 1996 burst) implies that something other than differential rotation (e.g., variation in the phase lag) must account for at least part of the observed frequency change. The prolonged decrease in frequency in the tail of the 31 December 1996 burst is not straightforwardly fit into this picture.

Despite these difficulties, the high stability (Strohmayer et al. 1998) and coherence (Markwardt \& Strohmayer 1999) of the brightness oscillations in the tails of bursts from sources such as $4 \mathrm{U}$ 1728-34 argue persuasively that the frequency in the tail of the bursts is close to either the fundamental or the first overtone of the neutron star spin frequency. Moreover, the general picture in which frequency changes are attributed to changes in the height of the emitting layer accounts approximately for the magnitude of the frequency change and explains why the frequency tends to rise near the beginning of the burst. However, in its current form it suffers from apparently serious problems. It is extremely important that there be a detailed investigation of, e.g., the coupling between differentially rotating layers, and that other ideas be explored so that the strengths and weaknesses of the rising layer model are put into sharper focus.

We thank Don Lamb and Fred Lamb for discussions about models of the frequency change, 
and Don Lamb, Dimitrios Psaltis, and Carlo Graziani for comments on a previous version of this paper. This research has made use of data obtained through the High Energy Astrophysics Science Archive Research Center Online Service, provided by the NASA/Goddard Space Flight Center. This work was supported in part by NASA grant NAG 5-2868, NASA AXAF contract SV 464006, and NASA ATP grant number NRA-98-03-ATP-028.

\section{REFERENCES}

Bildsten, L. 1995, ApJ, 438, 852

1998, in "The Many Faces of Neutron Stars", ed. R. Buccheri, A. Alpar, \& J. van Paradijs (Dordrecht: Kluwer), p. 419

Brown, E. F., \& Bildsten, L. 1998, ApJ, 496, 915

Buccheri, R. 1983, A\& A, 128, 245

Eadie, W. T., Drijard, D., James, F. E., Roos, M., \& Sadoulet, B. 1971, Statistical Methods in Experimental Physics (Amsterdam: North-Holland)

Fox, D. W., \& Lewin, W. H. G. 1999, IAUC 7081

Fryxell, B. A., \& Woosley, S. E. 1982, ApJ, 258, 773

Fushiki, I., \& Lamb, D. Q. 1987, ApJ, 323, L55

Helstrom, C. W. 1960, Statistical Theory of Signal Detection (New York: Pergamon)

Joss, P. C. 1978, ApJ, 225, L123

Lamb, D. Q., \& Melia, F. 1987a, ApJ, 321, L133

Leahy, D. A., Darbro, W., Elsner, R. F., Weisskopf, M. C., Sutherland, P. G., Kahn, S., \& Grindlay, J. E. 1983, ApJ, 266, 160

Livio, M., \& Bath, G. T. 1982, A\& A, 116, 286

Miller, M. C. 1999, ApJ, 515, L77

Nozakura, T., Ikeuchi, S., \& Fujimoto, M. Y. 1984, ApJ, 286, 221

Ruderman, M. 1981, Prog. Part. Nucl. Phys., 6, 215

Shara, M. M. 1982, ApJ, 261, 649

Strohmayer, T. E., \& Markwardt, C. B. 1999, ApJ Lett., accepted (astro-ph/9903062)

Strohmayer, T. E., Swank, J. H., \& Zhang, W. 1998a, in Proceedings of the Symposium "The Active X-Ray Sky: Results from BeppoSAX and Rossi-XTE", Rome, Italy, 21-24 October, 1997, Nuclear Physics B Proceedings Supplements. Eds. L. Scarsi, H. Bradt, P. Giommi, and F. Fiore

Strohmayer, T. E., Zhang, W., \& Swank, J. H. 1997, ApJ, 487, L77 
Strohmayer, T. E., Zhang, W., Swank, J. H., \& Lapidus, I. 1998b, ApJ, 503, L147

Strohmayer, T. E., Zhang, W., Swank, J. H., White, N. E., \& Lapidus, I. 1998c, ApJ, 493, L135 van Paradijs, J. et al. 1990, A\&A, 234, 181

Verbunt, F., \& van den Heuvel, E. P. J. 1995, in X-Ray Binaries, ed. W. H. G. Lewin, J. van Paradijs, \& E. P. J. van den Heuvel (Cambridge U. Press, Cambridge), 457

Wainstein, L. A., \& Zubakov, V. D. 1962, Extraction of Signals from Noise (Englewood Cliffs: Prentice-Hall) 